\title{
Protocol and Rationale: A 24-week Double-blind, Randomized, Placebo Controlled Trial of the Efficacy of Adjunctive Garcinia mangostana Linn. (Mangosteen) Pericarp for Schizophrenia
}

\author{
Alyna Turner ${ }^{1,2,3}$, John J. McGrath ${ }^{4,5,6}$, Olivia M. Dean ${ }^{1,3,7}$, Seetal Dodd ${ }^{1,3,8}$, Andrea Baker ${ }^{4}$, Susan M. Cotton ${ }^{8,9}$, \\ James G. Scott ${ }^{4,10,11}$, Bianca E. Kavanagh ${ }^{1}$, Melanie M. Ashton ${ }^{1,7,12}$, Adam J. Walker ${ }^{1}$, Ellie Brown ${ }^{1,8,9}$, \\ Michael Berk B,3,7,9 $^{1}$ \\ ${ }^{1}$ IMPACT Strategic Research Centre, School of Medicine, Deakin University, Barwon Health, Geelong, ${ }^{2}$ Faculty of Health and Medicine, School \\ of Medicine and Public Health, The University of Newcastle, Callaghan, ${ }^{3}$ Department of Psychiatry, University of Melbourne, Royal Melbourne \\ Hospital, Parkville, ${ }^{4}$ Queensland Centre for Mental Health Research, The Park Centre for Mental Health, Wacol, ${ }^{5}$ Queensland Brain Institute, \\ University of Queensland, St. Lucia, Australia, ${ }^{6}$ National Centre for Register-based Research, School of Business and Social Sciences, Aarhus \\ University, Aarhus, Denmark, ${ }^{7}$ Florey Institute of Neuroscience and Mental Health, University of Melbourne, Parkville, ${ }^{8}$ Centre for Youth Mental \\ Health, The University of Melbourne, Parkville, ${ }^{9}$ Orygen, The National Centre of Excellence in Youth Mental Health, Parkville, ${ }^{10}$ Metro North \\ Mental Health Service, ${ }^{11}$ Faculty of Medicine, The University of Queensland, Herston, ${ }^{12}$ Department of Psychiatry, University of Melbourne, \\ Professorial Unit, The Melbourne Clinic, Richmond, Australia
}

\begin{abstract}
Objective: Garcinia mangostana Linn., commonly known as mangosteen, is a tropical fruit with a thick pericarp rind containing bioactive compounds that may be beneficial as an adjunctive treatment for schizophrenia. The biological underpinnings of schizophrenia are believed to involve altered neurotransmission, inflammation, redox systems, mitochondrial dysfunction, and neurogenesis. Mangosteen pericarp contains xanthones which may target these biological pathways and improve symptoms; this is supported by preclinical evidence. Here we outline the protocol for a double-blind randomized placebo-controlled trial evaluating the efficacy of adjunctive mangosteen pericarp (1,000 mg/day), compared to placebo, in the treatment of schizophrenia.

Methods: We aim to recruit 150 participants across two sites (Geelong and Brisbane). Participants diagnosed with schizophrenia or schizoaffective disorder will be randomized to receive 24 weeks of either adjunctive 1,000 mg/day of mangosteen pericarp or matched placebo, in addition to their usual treatment. The primary outcome measure is mean change in the Positive and Negative Symptom Scale (total score) over the 24 weeks. Secondary outcomes include positive and negative symptoms, general psychopathology, clinical global severity and improvement, depressive symptoms, life satisfaction, functioning, participants reported overall improvement, substance use, cognition, safety and biological data. A 4-week post treatment interview at week 28 will explore post-discontinuations effects.

Results: Ethical and governance approvals were gained and the trial commenced.

Conclusion: A positive finding in this study has the potential to provide a new adjunctive treatment option for people with schizophrenia and schizoaffective disorder. It may also lead to a greater understanding of the pathophysiology of the disorder.
\end{abstract}

KEY WORDS: Mangosteen; Garcinia mangostana Linn.; Schizophrenia; Psychotic disorder; Treatment clinical trial; Oxidative stress.

\section{INTRODUCTION}

Received: September 7, 2018/ Revised: October 31, 2018

Accepted: November 1, 2018

Address for correspondence: Alyna Turner, $\mathrm{PhD}$

IMPACT Strategic Research Centre, P.O. Box 281, Geelong 3220,

VIC, Australia

Tel: +61-3-4215-3313, Fax: +61-3-4215-3491

E-mail: a.turner@deakin.edu.au

ORCID: https://orcid.org/0000-0001-7389-2546

\begin{abstract}
Background and Rationale
Current treatments for serious mental health conditions such as schizophrenia often leave patients with persistent symptoms, marked morbidity and substantially increased mortality risk. ${ }^{1)}$ Many antipsychotics used in the treatment
\end{abstract}

(ㄷ) This is an Open-Access article distributed under the terms of the Creative Commons Attribution Non-Commercial License (http://creativecommons.org/licenses/by-nc/4.0) which permits unrestricted non-commercial use, distribution, and reproduction in any medium, provided the original work is properly cited. 
of schizophrenia have limited therapeutic efficacy and are often associated with side-effects impacting patient health and quality of life. ${ }^{2)}$ The limitations of existing pharmacological treatments underline the urgent need for novel therapies for the treatment of schizophrenia. Specifically, new adjunctive treatments are needed to target alternate pathways concordant with the current understanding of the pathophysiology of schizophrenia. Several factors understood to contribute to the pathophysiology of schizophrenia may not be sufficiently targeted by conventional pharmacological therapies. These factors include oxidative stress, ${ }^{3-5)}$ increased inflammatory profiles, ${ }^{6}$ and changes to mitochondrial function and neurogenesis.

\section{Adjunctive Pharmacological Targets}

\section{Oxidative stress}

Considerable research indicates that oxidative stress is a contributor to the pathophysiology of schizophrenia. Lower levels of glutathione for example, which plays an important mitigating role in the redox process, have been observed in patients with schizophrenia. ${ }^{8,9)}$ Several treatments that act to modulate redox systems have been investigated as adjunctive options; including vitamin C (ascorbic acid), vitamin E ( $\alpha$-tocopherol), and $\alpha$-lipoic acid supplements among others (see reference ${ }^{8)}$ for review). These antioxidants indirectly target the primary redox system, promoting glutathione metabolism. Agents that directly target glutathione metabolism, such as $\mathrm{N}$-acetyl cysteine (NAC), have been shown to yield therapeutic benefit. ${ }^{8)}$ Improvements in negative symptoms were previously reported in two controlled trials of NAC for schizophrenia ${ }^{10,11)}$ (see reference ${ }^{12)}$ for review); and others are underway. ${ }^{13)}$

\section{Inflammatory pathways}

Inflammatory profile and immune dysfunction also appear to be significant factors in the pathophysiology of schizophrenia. According to a recent meta-analysis that included 18 schizophrenia studies, ${ }^{14)}$ elevated levels of the proinflammatory cytokines interleukin 6 (IL-6) and tumor necrosis factor alpha (TNF- $\alpha$ ), as well as the cytokine receptor antagonist IL-1Ra, and soluble cytokine receptor (sIL-2R) were associated with acute illness; while IL-6, IL-1 $\beta$ and sIL-2R levels were elevated in those patients deemed chronically ill. Interestingly, reductions in the levels of IL-6, sIL-2R were associated with acute treatment. ${ }^{14)}$ Despite current evidence, therapies that target inflammatory activity are underutilized, and limited immunomodulatory interventions for treatment of schizophrenia exist. ${ }^{14)}$ Notably, agents with immunomodulatory effects such as the tetracyclic antibiotic minocycline, and the cyclooxygenase 2 inhibitor celecoxib, have yielded promising results in the treatment of schizophrenia; however, currently available data are seemingly variable, and dependent on the stage of illness. ${ }^{15,16)}$

\section{Mitochondrial function}

Multiple lines of evidence also implicate mitochondrial dysfunction in the pathophysiology of schizophrenia. For example, an elevated risk of schizophrenia has been associated with a number of mitochondrial DNA single nucleotide polymorphisms. ${ }^{17)}$ Mitochondrial abundance, morphology, and functioning are also associated with illness onset and neuroprogression in schizophrenia ${ }^{18,19)}$ both in the brain ${ }^{20)}$ and the periphery. ${ }^{21,22)}$ While such deficits are not necessarily induced via treatment, it is important to note that a number of antipsychotics do have documented effects on mitochondrial function and metabolism. ${ }^{19)}$ Thus, mitigating wanton effects on mitochondrial function with appropriate agents is also desirable. Although several prospective mitochondrial agents have been evaluated in the context of other psychiatric disorders, ${ }^{23)}$ and the putative benefits of improving mitochondrial function using adjunctive agents-for example NAC and minocycline-has been discussed previously, ${ }^{19)}$ this therapeutic target is another currently underutilized.

\section{Neurogenesis and apoptotic pathways}

Other pathways that may be useful therapeutic targets for schizophrenia include neurotrophic and apoptotic pathways. Pathophysiological deficits in neuroprotection and neurogenesis may underlie the negative and cognitive symptoms observed in schizophrenia. ${ }^{24)}$ For example, significant reductions in cell proliferation $\left(\mathrm{Ki}^{+} 7^{+}\right.$cells) were recorded in the post-mortem hippocampal tissue of patients with schizophrenia. ${ }^{25,26)}$ Additionally, the dopaminergic deficits classically observed in schizophrenia have also been attributed to impaired neurogenesis in the striatum. ${ }^{27)}$ Some treatments, such as atypical antipsychotics, have been shown to promote hippocampal cell 
proliferation, though typical antipsychotics such as haloperidol (which has chiefly dopaminergic action) have neutral or even deleterious effects on neurogenesis. ${ }^{28,29)}$

Unsurprisingly, given the involvement of inflammatory cytokines, reactive oxidative species and mitochondrial dysfunction in the pathophysiology and neuroprogression of schizophrenia, irregularities in apoptotic and metabolic pathways are also observed. ${ }^{30)}$ Evidence indicates increases in apoptotic cell death pathways in the brains of patients with schizophrenia, for example in the anterior cingulate cortex and hippocampus. ${ }^{31)}$ In the periphery, elevated serum levels of the B cell lymphoma protein-2 (BCl-2), a regulator of cellular apoptosis, were previously associated with acute illness in patients with schizophrenia. ${ }^{32)}$ Also of note, levels of glycogen synthase kinase-3 $\beta$, which plays an important role in the PI3K/Akt/ mammalian target of rapamycin (mTOR) intracellular signalling pathway and a plethora of cellular processes including proliferation and apoptosis, were reduced in the platelets of schizophrenia patients. ${ }^{33)}$ Interestingly, this deficit improved following treatment with olanzapine, though it is unclear whether this was related to the antipsychotic effects of the drug. ${ }^{33)}$

In summary, oxidative stress, inflammation, altered neurogenesis and apoptosis are highly interdependent pathways, implicated in both the pathophysiology of schizophrenia and response to treatment. As such, it is reasonable to adduce that neuroprotective agents that act on these underutilized pathophysiological targets may offer considerable therapeutic value, particularly when offered as synergistic adjuncts. Accordingly, investigations of adjunctive agents with diverse therapeutic profiles are warranted.

\section{Garcinia mangostana Linn.}

Garcinia mangostana Linn., known colloquially as mangosteen, is a tropical evergreen fruit tree originating from Indonesia. The mangosteen fruit pericarp and exocarp both contain a number of bioactive compounds, including, but not limited to, polyphenols, such as xanthones and tannins, as well as anthocyanins, procyanins, prodelophinidins, and associated stereoisomers such as epicatechins. Over 85 metabolites have been isolated from the pericarp of mangosteen, which includes in excess of 60 xanthones. These include $\alpha$-mangostin, $\gamma$-mangostin, garcinone $E$, gartanin, 8-deoxygartanin, and 9-hydroxycalabaxanthone. ${ }^{34)}$

Alpha-mangostin increases glutathione peroxidase activity, a key enzyme in the glutathione antioxidant pathway. In addition, $\alpha$-mangostin influences apoptosis; it up-regulates mitogen activated protein kinase, extracellular signal-regulated kinases, and p53 cell signalling pathways, which have all been found to be altered in schizophrenia. ${ }^{35-37)}$ In animal models, xanthones from mangostin, including $\alpha$-mangostin have been found to be protective against iodoacetate-induced cell death ${ }^{38)}$ and doxorubicin induced central nervous system inflammatory toxicity. ${ }^{39)}$ Mangiferin, a glucoxixanthone, has been found to ameliorate 6-hydroxydopamine induced cytotoxicity and oxidative stress in the ketamine model of schizophrenia. Isogarcinol has anti-inflammatory activity through inhibition of calcineurin, a protein phosphatase that activates the $T$ cells of the immune system, decreasing delayed type hypersensitivity. Gartanin inhibits mTOR pathway, an important protein kinase that regulates cell growth, cell proliferation, cell motility, survival, protein synthesis, and transcription via Bcl-2 and p53, ${ }^{40,41)}$ two proteins that regulate cell death pathways. ${ }^{30)}$ Mangosteen pericarp added to the diet was shown to increase serum levels of brain-derived neurotrophic factorin a rodent model. ${ }^{42)}$

Recent preclinical research by Oberholzer et al. ${ }^{43)}$ investigated the effects of treatment with raw mangosteen rind on depression-like behaviors in Flinders Sensitive Line rats, using the novel object recognition and forced swim tests. Notably, mangosteen treatment reversed the cognitive deficits and depression-like behaviors phenotypic of this strain. In addition, mangosteen treatment also improved cortico-hippocampal deficits in 5-hydroxyindole acetic acid and serotonin turnover, as well as reversing lipid peroxidation in the hippocampus. ${ }^{43)}$ These preclinical findings both suggest potential mechanisms of action and applications potentially beneficial for other psychiatric disorders, including schizophrenia.

Taken together, the biological activities of mangosteen pericarp align with some of the alterations observed in schizophrenia. Given this, mangosteen pericarp may be an effective adjunctive therapy for the symptoms of schizophrenia.

\section{Objectives and Hypotheses}

The aim of this randomized, double-blind, place- 
bo-controlled trial is to determine if 24 weeks of adjunctive treatment with $1,000 \mathrm{mg} /$ day of mangosteen pericarp will improve clinical outcomes in people with schizophrenia (or schizoaffective disorder) compared to placebo.

\section{Primary hypothesis}

Adjunctive mangosteen pericarp treatment will be superior to placebo in the treatment of overall psychopathology in schizophrenia, as assessed by mean change in the total score of the Positive and Negative Syndrome Scale $\left(\right.$ PANSS $^{44)}$ ) total scores from baseline to week 24 (completion of the treatment phase and primary endpoint).

\section{Secondary hypotheses}

- Twenty-four weeks of adjunctive mangosteen pericarp treatment will be superior to placebo in reducing severity of general, positive and negative symptoms of schizophrenia (PANSS general, positive and negative subscale scores).

- Twenty-four weeks of adjunctive mangosteen pericarp treatment will be superior to placebo in reducing depression, substance use, and will improve functioning and quality of life.

- There will be no difference in adverse and serious adverse events (SAEs) between the two groups over the 24 weeks.

- Those taking adjunctive mangosteen pericarp treatments will have reduced levels of inflammatory and oxidative stress (lipid peroxidation) levels in peripheral (blood) samples compared to placebo over the 24 weeks.

- Changes in clinical and functioning measures over 24 weeks will correlate with measurable changes in inflammatory and oxidative markers in the adjunctive mangosteen pericarp group.

An exploratory aim is to delineate whether the improvements in response to mangosteen that are observed at 24-weeks, are sustained to the 28-weeks (4-weeks post-discontinuation).

\section{METHODS}

\section{Design and Setting}

This placebo-controlled randomized clinical trial is be- ing conducted in two sites in Australia; Geelong, Victoria, and Brisbane, Queensland. Ethics approval has been received from relevant committees, any protocol and forms amendments will be approved by the committees and all participants will provide informed consent prior to enrolment. The trial is being conducted in accordance with Good Clinical Practice (GCP) guidelines, Australian Clinical Trial guidelines, ${ }^{45)}$ and the National Statement on Ethical Conduct in Human Research. ${ }^{46)}$ The study protocol was developed in accordance with Standard Protocol Items Recommendations for Intervention Trials (SPIRIT) 2013 guidelines $^{47)}$ and prospectively registered with the Australian and New Zealand Clinical Trials Registry. Findings will be reported using Consolidated Standards of Reporting Trials (CONSORT) guidelines. ${ }^{48)}$

\section{Eligibility Criteria}

Inclusion criteria include: Being aged 18 years or over; meeting Diagnostic and Statistical Manual of Mental Disorders, 5th edition $\left(\mathrm{DSM}-5^{49)}\right.$ ) diagnostic criteria for schizophrenia or schizoaffective disorder; score $\geq 54$ on the PANSS and/or $\geq 3$ on the Clinical Global Impressions severity of illness scale $\left(\mathrm{CGI}-\mathrm{S}^{50}\right)$; have been on a stable psychotropic therapy regime for four weeks prior to study entry; be using effective contraception if female, sexually active and of childbearing age; be able to speak, read, write and understand the English language; have a current treating physician, and have capacity to consent to the study and comply with study procedures. If there is a delay of $>7$ days between screening and baseline assessments, or baseline assessment and medication commencement, the inclusion scales (PANSS and CGI-S) will be re-administered to ensure the participant still meets eligibility criteria.

Exclusion criteria include: Known or suspected clinically unstable systemic medical disorder; pregnancy or breastfeeding; contraindications or intolerance to mangosteen pericarp or any of the trial preparations; and/or current enrolment in another clinical trial.

Withdrawal criteria include: If a participant ceases taking their trial medication for seven consecutive days or if a participant ceases effective contraception or becomes pregnant. Participants will be withdrawn from the study if they withdraw consent or at the discretion of the researcher given adverse events or loss to follow-up. SAEs will not require automatic withdrawal from the study. 


\section{Interventions}

Mangosteen pericarp: One-thousand milligrams per day of mangosteen pericarp (given as two 500 mg capsules, once a day) will be used in the study. The dose and treatment duration was selected based on a pilot study in people with schizophrenia. ${ }^{51)}$ The investigational product (provided by VitalXan, Adelaide, Australia) is produced from mangosteen whole fruit. The fruit is cut and the fruit pulp is separated from the rind. The inner rind only, is extracted by water at $80^{\circ} \mathrm{C}$ for 2 to 3 hours. This is then evaporated and Brix determination (24 brix) is completed. The extract is sterilized $\left(90^{\circ} \mathrm{C}\right.$ for 30 minutes) and undergoes a spray drying process. The final product was analyzed for contaminants such as Escherichia coli, Salmonella and Clostridium.

Placebo: Placebo tablets are produced with identical capsules. Active and placebo capsules are identical in appearance, color and taste. Pharmaceutical Packaging Professionals utilized the randomization list to pack the capsules in identical bottles which were labelled with the anonymous Participant-ID.

The medication is dispensed on a four-weekly basis (baseline, weeks 4, 8, 12, 16 and 20), with the first bottle dispensed after the baseline visit has been completed and the participant has been randomized. Medication labelling complies with local regulatory GCP requirements and all investigational products kept in a secure location under appropriate storage conditions.

Participants are instructed to take two capsules once a day with food. They can select the time of day they choose to take the two capsules as long as it is consistent. Participants are instructed to return unused medication and adherence is assessed by pill counts of returned medication packs. Participants are regularly telephoned during the trial to enhance retention and medication adherence.

Concomitant care: Due to the adjunctive nature of the study, participants can stay on their current medications during the trial (medication regimes must have been stable for four weeks prior to randomization). Dose changes to existing medications (either increases or decreases in dose), or addition or removal of an agent will be accepted (with the exception of supplements containing mangosteen) and participants will be allowed to continue with the trial. All medications (prescribed, over the counter, and nutraceuticals/supplements) are recorded at baseline and monitored at study each visit with changes recorded.

\section{Outcomes}

\section{Primary outcome}

The primary outcome of the study is the change in PANSS total score from baseline to end treatment (week 24). The PANSS is a 30-item scale used to evaluate the presence and severity of positive symptoms ( 7 items), negative symptoms (7 items), and general psychopathology symptoms (16 items) of schizophrenia. ${ }^{44)}$ All items are rated on a 7-point scale (1=absent to $7=$ =xtreme), with detailed anchor points for each item. Each item is rated by the interviewer following a semi-structured interview (the SCI-PANSS), and for some items, collaborative information gathered from identified others (e.g., healthcare provider or carer). The PANSS is one of the most commonly used measures of psychopathology of schizophrenia, and has been rigorously tested for validity and reliability. ${ }^{52,53)}$

\section{Secondary outcomes}

Secondary outcomes include PANSS positive, negative and general subscales, overall symptom state both clinician-rated (CGI-S and CGI-global improvement [CGI-I]) and participant reported (Patient Global Improvement ${ }^{50)}$ ), depressive symptoms (Montgomery Åsberg Depression Rating Scale, MADRS ${ }^{54)}$ ), life satisfaction (Quality of Life Enjoyment and Satisfaction Questionnaire ${ }^{55)}$ ), functioning (Global Assessment of Functioning Scale, GAF ${ }^{56)}$; and the Longitudinal Interval Follow-up Evaluation-Range of Impaired Functioning Tool ${ }^{57)}$ ), cognition (CogState brief screen, assessing processing speed, attention, visual learning and working memory ${ }^{58)}$ ), substance use (Opiate Treatment Index, Alcohol Use Disorders Identification Test, Fagerstrom Test for Nicotine Dependence); and peripheral (blood) markers including markers of antioxidant defense (including antioxidant levels), oxidative stress (including markers of lipid peroxidation and protein carbonylation), and markers of inflammation (including IL-1, IL-6, C-reactive protein, and TNF- $\alpha$ ) and a range of other sera markers related to general health, which interact with oxidative stress.

\section{Other outcomes}

Weight, body mass index, medication use.

\section{Additional baseline measures}

Demographics; diagnosis (schizophrenia or schizoaffective according to the Diagnostic Interview for Psychosis, ${ }^{59)}$ 
using a diagnostic crosswalk to align diagnoses with DSM-5); mental health history; height and physical activity (International Physical Activity Questionnaire ${ }^{60)}$ ). Time points for measurement can be seen in Figure 1.

\section{Participant Timeline}

Following referral to the study, trial clinicians will contact the potential participant and schedule an initial face-to-face screening interview. During the screening interview, written informed consent will be provided, and eligibility determined. Competency to provide informed consent will be determined by the interviewer in collaboration with the treating physician if required. Once eligibility is determined, participants will complete the baseline assessment, randomized (using the next consecutive participant number for that site), provide the baseline blood sample and then be provided with 4 weeks of medication and instructions for use. Every 4 weeks the participants will return for a face-to-face interview, will return any remaining medication in the bottle, and be provided

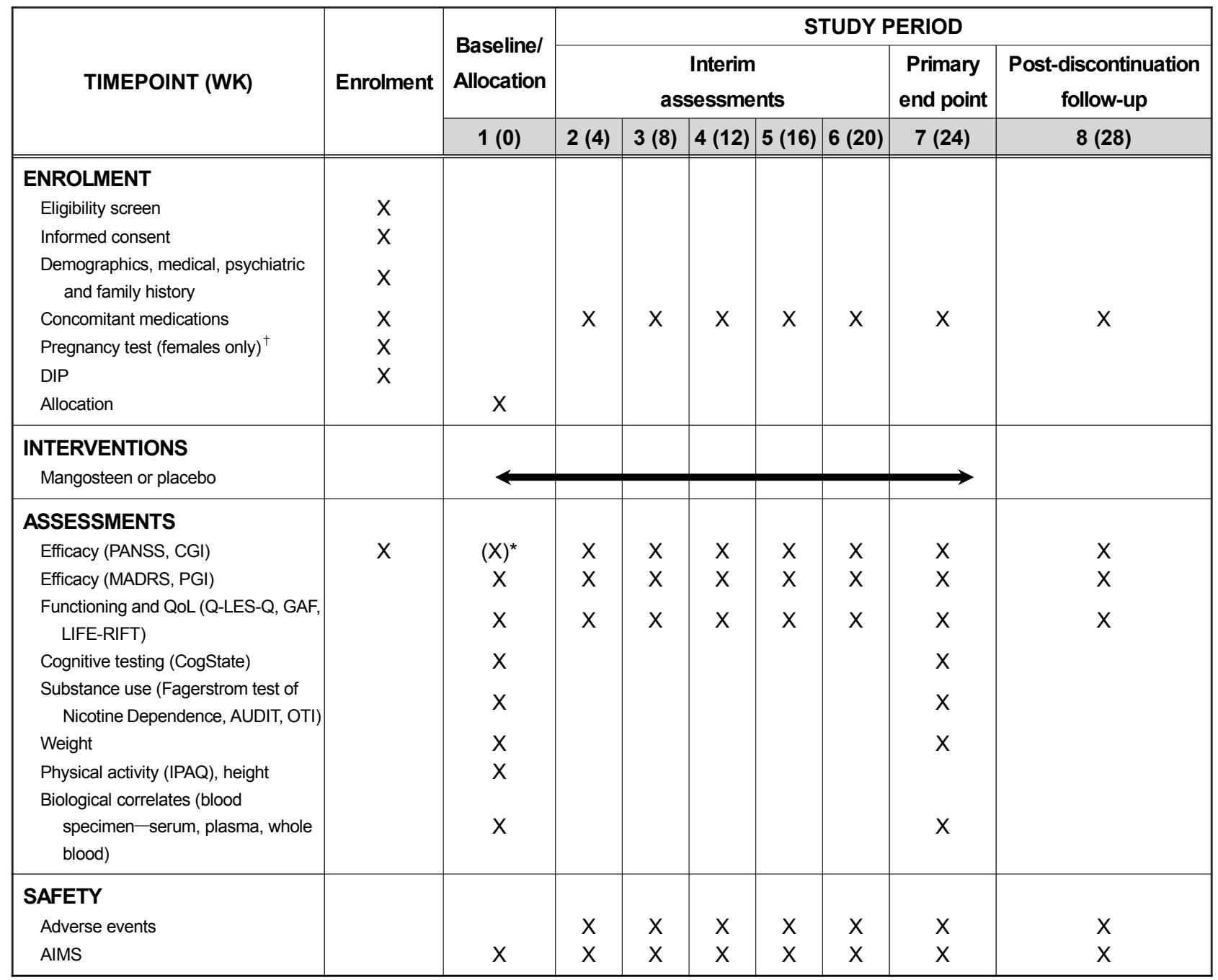

Fig. 1. Trial schedule of enrolment, interventions, and assessments.

DIP, Diagnostic Interview for Psychosis; PANSS, Positive and Negative Syndrome Scale; CGI, Clinical Global Impressions; MADRS, Montgomery Åsberg Depression Rating Scale; PGI, Patient Global Improvement; QoL, quality of life; Q-LES-Q, Quality of Life Enjoyment and Satisfaction Questionnaire; GAF, Global Assessment of Functioning Scale; LIFE-RIFT, Longitudinal Interval Follow-up Evaluation-Range of Impaired Functioning Tool; AUDIT, Alcohol Use Disorders Identification; OTI, Opiate Treatment Index; IPAQ, International Physical Activity Questionnaire Test; AIMS, Abnormal Involuntary Movement Scale.

*PANSS and CGI repeated if $>7$ days between screening and baseline assessments.

${ }^{\dagger}$ If participant is not sexually active or the treating clinician feels it is not warranted, this is not compulsory. 
with new medications (weeks 4, 8, 12, 16, 20). At week 24 the participant will complete the face-to-face primary end-point interview, including providing the endpoint blood sample. A final post-discontinuation visit will be held at week 28 (Fig. 1).

\section{Recruitment}

Clinicians at both Geelong and Brisbane sites will recruit from mental health services in the surrounding districts. In addition, the Geelong site will utilize community recruitment, utilizing strategies including advertisement through local newspapers, the internet and radio, flyers in medical waiting rooms, pharmacies and university campuses, and direct contact with other potential referral sources (general practitioners, the disability support sector, support groups, and private psychiatrists). Participants will be reimbursed for their travel costs to a total of $20 \mathrm{AUD} / \mathrm{visit}$.

\section{Assignment of Interventions}

Following the baseline assessment, participants are randomized to either the mangosteen group or the placebo group. Allocation to treatment arm is randomly assigned in a 1:1 ratio (active to placebo) using permutated block randomization, stratified by site (Brisbane and Geelong). An independent researcher developed a computer-generated randomization plan using the website randomization.com. Participants, trial clinicians, investigators, and statisticians were blinded to treatment allocation.

Copies of the randomization list are held by the clinical trials pharmacy in case of requests for unblinding, with a copy kept in a sealed envelope by the investigators in case of emergency. All cases of emergency unblinding will be thoroughly documented and require reporting to the Data Safety and Monitoring Board (DSMB). Participants will be notified which arm of the study they took part in after the completion of all participants in the study.

\section{Data Collection and Management}

Trial data will be collected during face-to-face interviews from active participants. For participants who withdraw or are withdrawn by the investigator, a post-withdrawal visit will be conducted where possible, and thereafter no further information will be collected. Trial clinicians will be trained in outcomes measures with in- ter-rater reliability assessments regularly conducted within and across sites. Paper case report forms will be utilized during the interviews, and trial clinicians across both sites will enter data into an electronic database using the REDCap system, ${ }^{61)}$ a metadata driven internet browser-based system for data management. Paper and electronic trial data will be stored de-identified and kept secure and confidential. Identifying participant information will be stored separately from trial data.

For consenting participants, blood samples will be obtained at baseline and week 24. Paxgene or whole blood, serum and plasma aliquots will be labelled with participant ID and stored in $-80^{\circ} \mathrm{C}$ freezer until analysis.

\section{Sample Size}

We have pilot data ${ }^{51)}$ on 80 individuals with schizophrenia who received 1,000 mg of mangosteen pericarp for 180 days. In the pilot data, the effect size on the primary outcome of the PANSS total was large (Cohen's $d=1.41$ ). For the current study, it was important to consider that effect sizes are typically smaller in follow up studies than in pilot studies, enabling the study to detect a difference between groups of both clinical and scientific interest. Therefore, conservative power estimations for the current study were made based on the smallest effect observed in the pilot study (i.e., medium effect for GAF). With alpha $(\alpha)$ at the 0.05 level, a sample size of 50 per group or a total sample size of 100 would provide $90 \%$ power $(1-\beta)$ to detect a significant difference. A sample size of 150 (75 per group), will allow for attrition rates of up to $50 \%{ }^{62)}$

\section{Statistical Methods}

All analyses will be conducted in accordance with the International Conference on Harmonization E9 statistical principles. Analysis will be based on all randomized participants with at least one post-baseline observation and is therefore modified-intention-to-treat analysis. The primary efficacy analysis will assess average treatment group differences for the primary outcome measure PANSS total score over the entire treatment period and will use a likelihood based mixed-effects model, repeated measures approach (MMRM). The MMRM model includes the fixed, categorical effects of treatment, visit, and treatment-byvisit interaction, as well as the continuous, fixed covariates of baseline score and baseline score-by-visit 
interaction. Planned comparisons will be done with the MMRM models to determine between group differences in change of symptoms measures from baseline to week 24, baseline to week 28 and week 24 to week 28 . Analyses of dichotomous data will be presented as proportions, with 95\% confidence interval, and Fisher's exact $p$ value where appropriate. Non-parametric statistics will be used when assumptions for parametric methods are violated. Effect sizes will be calculated using Cohen's guidelines. All tests of treatment effects will be conducted using a two-sided alpha level of 0.05 and 95\% confidence intervals. Change scores in symptoms and in biological markers will be derived. Pearson Product Moment Correlations $(r)$ will be used to examine the relationships between the change scores for symptoms and the change scores for biological markers in the two groups. The correlations obtained for each group will be tested using Fisher's z transformation to determine if they are statistically different in terms of strength.

\section{Monitoring and Harms}

The study is being sponsored by Barwon Health and the University of Queensland and has been funded by the Stanley Medical Research Institute

A DSMB has been established for the trial, consisting of members with experience in clinical trials pharmacy, psychiatry, and statistics. The DSMB will review aggregate and individual subject safety data and provide recommendations to continue or terminate the trial depending on this data, reported to the principal investigators.

All adverse events (including SAEs) will be recorded, intervened according to medical assessment, and monitored. Participants are asked open-ended questions about how they have been feeling in general and if they have any concerns regarding their health or the study medication. Reporting of SAEs to regulatory authorities will be done by the investigator (or delegate) in accordance with local regulations. The Abnormal Involuntary Movement Scale (AIMS), a standard rating scale for neurolepticbased clinical trials, will also be administered at every interview. If the participant is of child-bearing potential and sexually active, urine pregnancy tests will be conducted prior to randomization. All participants will be required to nominate a current treating physician on enrolment into the study and will be notified that that physician may be contacted, should the research team feel it is ap- propriate, for example if the participant expresses suicidal ideation with a specific plan or intent (a MADRS suicide item score of 5 or 6 ).

\section{Ancillary and post-trial care}

Participants will be provided with details on how the product can be purchased following completion in the study. If a participant suffers an injury as a result of participation and seeks compensation, the trial sponsor will comply with the process and conditions set out in the Medicines Australia Guidelines for Compensation for Injury Resulting from Participation in a Clinical Trial.

\section{Dissemination Policy}

Participants will be provided with a summary of results after analysis is complete. Final results will be published after completion of the study. De-identified data will be submitted and shared through the National Institute of Mental Health Data Archive (NDA). Data will be submitted to the National Database for Clinical Trials system related to Mental Illness which is part of the NDA.

\section{RESULTS}

Ethical and governance approvals were gained and recruitment of participants commenced. The trial has received ethics approval from Barwon Health Human Research and Ethics Committee and Metro South Human Research Ethics Committee.

\section{DISCUSSION}

There is a need for new pharmacological treatments for people with schizophrenia that target alternate mechanisms of action to current treatment options. There is evidence of anomalies in redox biology and inflammation in schizophrenia, including the presence of oxidative stress particularly implicating the glutathione system; changes in oxidative status with treatment; and evidence that other glutathione and redox active agents have therapeutic value. Preclinical evidence suggests a range of compounds in mangosteen may directly address these anomalies and efficacy should be investigated in a well powered trial. The availability of a novel and tolerable agent for this disabling disorder would have a major international impact on health outcomes. As mangosteen is relatively af- 
fordable and accessible, evidence of efficacy could then be followed with an immediate roll out of the treatment.

\section{- Acknowledgments}

This study is being funded by the Stanley Medical Research Institute. All authors gratefully acknowledge their support.

Alyna Turner has received travel or grant support from the National Stroke Foundation, Hunter Medical Research Institute, Helen Macpherson Smith Trust, Schizophrenia Fellowship NSW, SMHR, ISAD, and the University of Newcastle. John J. McGrath is supported by grant APP1056929 from the John Cade Fellowship from the National Health and Medical Research Council. Olivia M. Dean is a R.D. Wright Biomedical Research Fellow and has received grant support from the Brain and Behavior Foundation, Simons Autism Foundation, Stanley Medical Research Institute, Deakin University, Lilly, NHMRC and ASBDD/Servier. She has also received in kind support from BioMedica Nutracuticals, NutritionCare and Bioceuticals. Olivia M. Dean gratefully acknowledges the support of the NHMRC (APP1145634). Seetal Dodd has received grant support from Stanley Medical Research Institute, NHMRC, Beyond Blue, ARHRF, Simons Foundation, Geelong Medical Research Foundation, Fondation FondaMental, Eli Lilly, Glaxo SmithKline, Organon, Mayne Pharma and Servier. He has received speaker's fees from Eli Lilly, advisory board fees from Eli Lilly and Novartis and conference travel support from Servier. Andrea Baker reports no disclosures. Susan M. Cotton has received grant support from the NHMRC, the Stanley Medical Research Institute, BeyondBlue, Movember, The University of Melbourne, Australian Catholic University, ARHRF, and Mental Illness Research Fund (Victoria Department of Human Services). She is supported by a NHMRC Senior Research Fellowship (APP1136344). James G. Scott is supported by a National Health and Medical Research Council Practitioner Fellowship Grant (APP1105807), has been a speaker for Janssen Cilag, Lundbeck, Servier, and Shire Pharmaceuticals, and served as a consultant to Janssen Cilag, Lundbeck and Roche. Bianca E. Kavanagh has received research support from the Australian Government Research Training Program Scholarship, and lan Scott Mental Health PhD Scholarship, Australian Rotary Health. Melanie M. Ashton has received grant/research support from Deakin University,
Australasian Society for Bipolar Depressive Disorders, Lundbeck, Australian Rotary Health, lan Parker Bipolar Research Fund, Cooperative Research Centre for Mental Health and PDG Geoff and Betty Betts Award from Rotary Club of Geelong. Adam J. Walker was previously supported by an Australian Postgraduate Award and an HDR Writing Award from Deakin University. Ellie Brown has received travel and grant support from the University of the West of England, UK. Michael Berk has received grant support from NIH, Simons Autism Foundation, Cancer Council of Victoria, CRC for Mental Health, Stanley Medical Research Foundation, MBF, NHMRC, Beyond Blue, Geelong Medical Research Foundation, Bristol Myers Squibb, Eli Lilly, GlaxoSmithKline, Organon, Novartis, Mayne Pharma and Servier. Michael Berk has been a speaker for Astra Zeneca, Bristol Myers Squibb, Eli Lilly, GlaxoSmithKline, Janssen Cilag, Lundbeck, Merck, Pfizer, Sanofi Synthelabo, Servier, Solvay and Wyeth, and served as a consultant to Astra Zeneca, Bristol Myers Squibb, Eli Lilly, GlaxoSmithKline, Janssen Cilag, Lundbeck and Servier. Michael Berk is a co-inventor on two provisional patents regarding the use of NAC and related compounds for psychiatric indications, assigned to the Mental Health Research Institute.

\section{REFERENCES}

1. Ferrari AJ, Saha S, McGrath JJ, Norman R, Baxter AJ, Vos T, et al. Health states for schizophrenia and bipolar disorder within the Global Burden of Disease 2010 study. Popul Health Metr 2012;10:16.

2. Miyamoto S, Duncan GE, Marx CE, Lieberman JA. Treatments for schizophrenia: a critical review of pharmacology and mechanisms of action of antipsychotic drugs. Mol Psychiatry 2005;10:79-104.

3. Dean OM, van den Buuse M, Bush Al, Copolov DL, Ng F, Dodd $\mathrm{S}$, et al. A role for g/utathione in the pathophysiology of bipolar disorder and schizophrenia? Animal models and relevance to clinical practice. Curr Med Chem 2009;16:29652976.

4. Flatow J, Buckley P, Miller BJ. Meta-analysis of oxidative stress in schizophrenia. Biol Psychiatry 2013;74:400-409.

5. Ng F, Berk M, Dean O, Bush Al. Oxidative stress in psychiatric disorders: evidence base and therapeutic implications. Int J Neuropsychopharmacol 2008;11:851-876.

6. Leboyer M, Berk M, Yolken RH, Tamouza R, Kupfer D, Groc L. Immuno-psychiatry: an agenda for clinical practice and innovative research. BMC Med 2016;14:173.

7. Davis J, Eyre H, Jacka FN, Dodd S, Dean O, McEwen S, et al. A review of vulnerability and risks for schizophrenia: beyond 
the two hit hypothesis. Neurosci Biobehav Rev 2016;65:185-194.

8. Magalhães PV, Dean O, Andreazza AC, Berk M, Kapczinski F. Antioxidant treatments for schizophrenia. Cochrane Database Syst Rev 2016;2:CD008919.

9. Wood SJ, Yücel M, Pantelis C, Berk M. Neurobiology of schizophrenia spectrum disorders: the role of oxidative stress. Ann Acad Med Singapore 2009;38:396-401.

10. Berk M, Copolov D, Dean O, Lu K, Jeavons S, Schapkaitz I, et al. N-acetyl cysteine as a g/utathione precursor for schizophrenia--a double-blind, randomized, placebo-controlled trial. Biological Psychiatry. 2008;64:361-368.

11. Farokhnia M, Azarkolah A, Adinehfar F, Khodaie-Ardakani MR, Hosseini SM, Yekehtaz $\mathrm{H}$, et al. N-acetylcysteine as an adjunct to risperidone for treatment of negative symptoms in patients with chronic schizophrenia: a randomized, double-blind, placebo-controlled study. Clin Neuropharmacol 2013;36:185-192.

12. Deepmala, Slattery J, Kumar N, Delhey L, Berk M, Dean O, et al. Clinical trials of $\mathrm{N}$-acety/cysteine in psychiatry and neurology: a systematic review. Neurosci Biobehav Rev 2015;55: 294-321.

13. Rossell SL, Francis PS, Galletly C, Harris A, Siskind D, Berk M, et al. N-acetylcysteine (NAC) in schizophrenia resistant to clozapine: a double blind randomised placebo controlled trial targeting negative symptoms. BMC Psychiatry 2016;16:320.

14. Goldsmith DR, Rapaport MH, Miller BJ. A meta-analysis of blood cytokine network alterations in psychiatric patients: comparisons between schizophrenia, bipolar disorder and depression. Mol Psychiatry 2016;21:1696-1709.

15. Müller N. Immunological aspects of the treatment of depression and schizophrenia. Dialogues Clin Neurosci 2017;19: 55-63.

16. Sommer IE, van Westrhenen R, Begemann MJ, de Witte LD, Leucht S, Kahn RS. Efficacy of anti-inflammatory agents to improve symptoms in patients with schizophrenia: an update. Schizophr Bull 2014;40:181-191.

17. Flippo KH, Strack S. An emerging role for mitochondrial dynamics in schizophrenia. Schizophr Res 2017;187:26-32.

18. Konradi C, Öngür D. Role of mitochondria and energy metabolism in schizophrenia and psychotic disorders. Schizophr Res 2017;187:1-2.

19. Rajasekaran A, Venkatasubramanian G, Berk M, Debnath M. Mitochondrial dysfunction in schizophrenia: pathways, mechanisms and implications. Neurosci Biobehav Rev 2015; 48:10-21.

20. Roberts RC. Postmortem studies on mitochondria in schizophrenia. Schizophr Res 2017;187:17-25.

21. Inuwa IM, Peet M, Williams MA. QSAR modeling and transmission electron microscopy stereology of altered mitochondrial ultrastructure of white blood cells in patients diagnosed as schizophrenic and treated with antipsychotic drugs. Biotech Histochem 2005;80:133-137.

22. Uranova N, Bonartsev P, Brusov O, Morozova M, Rachmanova
$\mathrm{V}$, Orlovskaya D. The ultrastructure of lymphocytes in schizophrenia. World J Biol Psychiatry 2007;8:30-37.

23. Pereira C, Chavarria V, Vian J, Ashton MM, Berk M, Marx W, et al. Mitochondrial agents for bipolar disorder. Int $)$ Neuropsychopharmacol 2018;21:550-569.

24. Kim YK, Na KS. Neuroprotection in schizophrenia and its therapeutic implications. Psychiatry Investig 2017;14:383391.

25. Allen KM, Fung SJ, Weickert CS. Cell proliferation is reduced in the hippocampus in schizophrenia. Aust N Z J Psychiatry 2016;50:473-480.

26. Reif A, Fritzen S, Finger M, Strobel A, Lauer M, Schmitt A, et al. Neural stem cell proliferation is decreased in schizophrenia, but not in depression. Mol Psychiatry 2006; 11:514522.

27. Inta D, Lang UE, Borgwardt S, Meyer-Lindenberg A, Gass P. Microglia activation and schizophrenia: lessons from the effects of minocycline on postnatal neurogenesis, neuronal survival and synaptic pruning. Schizophr Bull 2017;43:493-496.

28. Chikama K, Yamada H, Tsukamoto T, Kajitani K, Nakabeppu Y, Uchimura N. Chronic atypical antipsychotics, but not haloperidol, increase neurogenesis in the hippocampus of adult mouse. Brain Res 2017;1676:77-82.

29. Halim ND, Weickert CS, McClintock BW, Weinberger DR, Lipska BK. Effects of chronic haloperidol and clozapine treatment on neurogenesis in the adult rat hippocampus. Neuropsychopharmacology 2004;29:1063-1069.

30. Morris G, Walker AJ, Berk M, Maes M, Puri BK. Cell death pathways: a novel therapeutic approach for neuroscientists. Mol Neurobiol 2018;55:5767-5786.

31. Boiadzhian AS, Chavushian AC, Zakharian RV, Mkrtchian GM. Markers of apoptotic dysfunction in schizophrenia. Mol Biol 2013;47:674-680.

32. Tsai MC, Liou CW, Lin TK, Lin IM, Huang TL. BCl-2 associated with positive symptoms of schizophrenic patients in an acute phase. Psychiatry Res 2013;210:735-738.

33. Ferreira AS, Raposo NR, Sallet PC, Van de Bilt MT, Machado-Vieira R, Talib LL, et al. Lower phosphorylated glycogen synthase kinase-3B levels in platelets of patients with schizophrenia: increment by olanzapine treatment. Eur Arch Psychiatry Clin Neurosci 2015;265:167-170.

34. Pedraza-Chaverri J, Cárdenas-Rodríguez N, Orozco-lbarra M, Pérez-Rojas JM. Medicinal properties of mangosteen (Garcinia mangostana). Food Chem Toxicol 2008;46:32273239.

35. Funk AJ, McCullumsmith RE, Haroutunian V, Meador-Woodruff $\mathrm{JH}$. Abnormal activity of the MAPK- and CAMP-associated signaling pathways in frontal cortical areas in postmortem brain in schizophrenia. Neuropsychopharmacology 2012;37:896905.

36. Ni X, Trakalo J, Valente J, Azevedo MH, Pato MT, Pato CN, et al. Human p53 tumor suppressor gene (TP53) and schizophrenia: case-control and family studies. Neurosci Lett 2005; 
388:173-178.

37. Pereira A, Sugiharto-Winarno A, Zhang B, Malcolm P, Fink G, Sundram S. Clozapine induction of ERK1/2 cell signalling via the EGF receptor in mouse prefrontal cortex and striatum is distinct from other antipsychotic drugs. Int I Neuropsychopharmacol 2012;15:1149-1160.

38. Reyes-Fermín LM, González-Reyes S, Tarco-Álvarez NG, Hernández-Nava M, Orozco-lbarra M, Pedraza-Chaverri J. Neuroprotective effect of $\alpha$-mangostin and curcumin against iodoacetate-induced cell death. Nutr Neurosci 2012;15:34-41.

39. Tangpong J, Miriyala S, Noel T, Sinthupibulyakit C, Jungsuwadee P, St Clair DK. Doxorubicin-induced central nervous system toxicity and protection by xanthone derivative of Garcinia mangostana. Neuroscience 2011;175: 292-299.

40. Li G, Thomas S, Johnson JJ. Polyphenols from the mangosteen (Garcinia mangostana) fruit for breast and prostate cancer. Front Pharmacol 2013;4:80.

41. Liu Z, Antalek M, Nguyen L, Li X, Tian X, Le A, et al. The effect of gartanin, a naturally occurring xanthone in mangosteen juice, on the mTOR pathway, autophagy, apoptosis, and the growth of human urinary bladder cancer cell lines. Nutr Cancer 2013;65 Supp/ 1:68-77.

42. Huang HJ, Chen WL, Hsieh RH, Hsieh-Li HM. Multifunctional effects of mangosteen pericarp on cognition in C57BL/6J and triple transgenic Alzheimer's mice. Evid Based Complement Alternat Med 2014;2014:813672.

43. Oberholzer I, Möller M, Holland B, Dean OM, Berk M, Harvey BH. Garcinia mangostana Linn displays antidepressant-like and pro-cognitive effects in a genetic animal model of depression: a bio-behavioral study in the Flinders Sensitive Line rat. Metab Brain Dis 2018;33:467-480.

44. Kay SR, Fiszbein A, Opler LA. The Positive and Negative Syndrome Scale (PANSS) for schizophrenia. Schizophr Bull 1987;13:261-276.

45. Therapeutic Goods Administration. The Australian clinical trial handbook. Canberra:Department of Health and Ageing, Australian Government;2006.

46. National Health and Medical Research Council, Australian Research Council, Australian Vice-Chancellors' Committee. National Statement on Ethical Conduct in Human Research 2007 (updated May 2015). Canberra:National Health and Medical Research Council, Australian Government;2007.

47. Chan AW, Tetzlaff JM, Altman DG, Laupacis A, Gøtzsche PC, Krleža-Jerić K, et al. SPIRIT 2013 statement: defining standard protocol items for clinical trials. Ann Int Med 2013;158: 200-207.

48. Schulz KF, Altman DG, Moher D; CONSORT Group. CONSORT 2010 statement: updated guidelines for reporting parallel group randomised trials. BMJ 2010;340:c332.

49. American Psychiatric Association. Diagnostic and statistical manual of mental disorders. 5th ed. Washington,
DC:American Psychiatric Association;2013.

50. Guy W. ECDEU Assessment Manual for Psychopharmacology. Rockville, MD:U.S. Department of Health, Education, and Welfare; 1976.

51. Laupu WK. Efficacy of Garcinia mangostana L. (mangosteen) pericarp as an adjunctive to second-generation antipsychotics for the treatment of schizophrenia: a double blind, randomised, placebo-controlled trial. Townsville:James Cook University;2014. [Dissertation]

52. Kay SR, Opler LA, Lindenmayer JP. The Positive and Negative Syndrome Scale (PANSS): rationale and standardisation. Br J Psychiatry Suppl 1989:59-67.

53. Peralta V, Cuesta MJ. Psychometric properties of the Positive and Negative Syndrome Scale (PANSS) in schizophrenia. Psychiatry Res 1994;53:31-40.

54. Montgomery SA, Asberg M. A new depression scale designed to be sensitive to change. Br J Psychiatry 1979;134:382-389.

55. Endicott J, Nee J, Harrison W, Blumenthal R. Quality of life enjoyment and satisfaction questionnaire: a new measure. Psychopharmacol Bull 1993;29:321-326.

56. American Psychiatric Association. Diagnostic and statistical manual of mental disorders: primary care version. 4th ed. Washington, DC:American Psychiatric Association; 1994.

57. Leon AC, Solomon DA, Mueller TI, Endicott J, Posternak M, Judd LL, et al. A brief assessment of psychosocial functioning of subjects with bipolar I disorder: the LIFE-RIFT. Longitudinal interval follow-up evaluation-range impaired functioning tool. J Nerv Ment Dis 2000;188:805-812.

58. Maruff P, Thomas E, Cysique L, Brew B, Collie A, Snyder P, et al. Validity of the CogState brief battery: relationship to standardized tests and sensitivity to cognitive impairment in mild traumatic brain injury, schizophrenia, and AIDS dementia complex. Arch Clin Neuropsychol 2009;24:165-178.

59. Castle DJ, Jablensky A, McGrath JJ, Carr V, Morgan V, Waterreus A, et al. The diagnostic interview for psychoses (DIP): development, reliability and applications. Psychol Med 2006:36:69-80.

60. Craig CL, Marshall AL, Sjöström M, Bauman AE, Booth ML, Ainsworth $\mathrm{BE}$, et al. International physical activity questionnaire: 12-country reliability and validity. Med Sci Sports Exerc 2003;35:1381-1395.

61. Harris PA, Taylor R, Thielke R, Payne J, Gonzalez N, Conde JG. Research electronic data capture (REDCap)--a metadata-driven methodology and workflow process for providing translational research informatics support. J Biomed Inform 2009;42:377-381.

62. Kemmler G, Hummer M, Widschwendter C, Fleischhacker WW. Dropout rates in placebo-controlled and active-control clinical trials of antipsychotic drugs: a meta-analysis. Arch Gen Psychiatry 2005;62:1305-1312. 